\title{
Some Remarks on the Relation between Annotated Ordered Sets and Pattern Structures
}

\author{
Tim B. Kaiser ${ }^{1}$ and Stefan E. Schmidt ${ }^{2}$ \\ 1 SAP AG, Walldorf \\ 2 Institut für Algebra, Technische Universität Dresden
}

\begin{abstract}
We exhibit an intimate connection between the concept of an annotated ordered set and that of a pattern structure. This enables an exchange of ideas and techniques between both domains.
\end{abstract}

\section{Introduction}

Pattern structures were introduced in KG01 to model information. The usefulness of annotated ordered sets for similar purposes was studied in KSJ08. Here, we compare and relate the two approaches. To keep the article rationably selfcontained we recapitulate both concepts. We see how theorems can be moved between both domains and fix a theorem from KG01.

In the first section we will introduce annotated ordered sets. In the second section we will outline pattern structures. In the third section we will argue that pattern structures can be understood as a certain sub-class of annotated ordered sets and review basic constructions and theorems. In the fourth section we will correct a theorem about pattern structures and their projections.

The reader is assumed to be knowledgeable about the basics of order theory and formal concept analysis as can be found in DP90] and GW99.

\section{Annotated Ordered Sets}

Annotated ordered sets were introduced to model taxonomies, e.g. appearing in the realm of the Gene Ontology.

Definition 1 ((elementary) annotated ordered set). Let $\mathcal{P}:=\left(P, \leq_{\mathcal{P}}\right)$ be a finite ordered set (poset), let $X$ be a finite set of labels, and let $F: X \rightarrow 2^{P}$ be an annotation function. Then we call $\mathbb{O}:=(\mathcal{P}, X, F)$ an annotated ordered set and refer to $(X, F)$ as an annotation of $\mathcal{P}$. In case $\mathcal{P}$ is a (complete) lattice we call $\mathbb{O}$ an annotated (complete) lattice denoted $\mathbb{L}$. If $|F(x)|=1$ for all $x \in X$, for convenience, we regard $F$ as a map from $X$ to $P$ and say that $\mathbb{O}$ is elementary.

It is interesting to note that an annotated ordered set $(\mathcal{P}, X, F)$ can be regarded as a formal context with ordered attributes $\left(X, \mathcal{P}, F_{R}\right)$ since every mapping $T: A \rightarrow 2^{B}$ can be interpreted as a relation $T_{R} \subseteq A \times B$ where $x T_{R} y$ if and only if $y \in T(x)$. But the concept lattice of this formal context would not yield the 
intended concept lattice representation of the annotated ordered set, since it does not express the semantics of annotation in a taxonomy, that is, if we annotate an example to a class in a taxonomy we implicitly mean that it belongs to all the super-classes of the class it was annotated to. Additionally, modelling the annotated taxonomy as formal context would be rather counter-intuitive.

We describe how an appropriate formal context can be derived from an annotated ordered set to produce its concept lattice representation. For an ordered set $\mathcal{P}:=\left(P, \leq_{\mathcal{P}}\right)$ and node $q \in P$ we denote by $\uparrow q:=\left\{p \in P \mid q \leq_{\mathcal{P}} p\right\}$ the principal filter of $q$ and dually by $\downarrow q$ the principal ideal. Also, for later use, for $N \subseteq P$ let $\downarrow_{N} q:=\left\{n \in N \mid n \leq_{\mathcal{P}} q\right\}$ and dually $\uparrow_{N} q:=\left\{n \in N \mid q \leq_{\mathcal{P}} n\right\}$. Given an annotated ordered set $\mathbb{O}:=(\mathcal{P}, X, F)$ we can construct a formal context $\mathbb{K}_{\mathbb{O}}:=$ $(X, P, I)$ where we define the relation $I \subseteq X \times P$ by $x I p: \Longleftrightarrow \downarrow p \cap F(x) \neq \emptyset$.

This means that $I$ equals $F_{R} \circ \leq_{\mathcal{P}}$. Note that in the case of an elementary annotated ordered set the definition of $I$ can be written as $x I p: \Longleftrightarrow F(x) \leq p$ since $F$ is regarded as mapping to $P$ (instead of $2^{P}$ ).

The concept lattice of $\mathbb{K}_{\mathbb{O}}$ will be denoted by $\underline{\mathfrak{B}}_{\mathbb{O}}:=\left(\mathfrak{B}_{\mathbb{O}}, \leq_{\mathfrak{B}_{\mathbb{O}}}\right)$, where $\mathfrak{B}_{\mathbb{O}}:=$ $\mathfrak{B}\left(\mathbb{K}_{\mathbb{O}}\right)$ is the set of formal concepts of the formal context $\mathbb{K}_{\mathbb{O}}$ GW99. $\underline{\mathfrak{B}}_{\mathbb{O}}$ is called the concept lattice representation of the annotated ordered set $\mathbb{O}$.

In case $\mathbb{O}$ forms an annotated complete lattice and $(A, B) \in \mathfrak{B}_{\mathbb{O}}$ is a formal concept in $\mathfrak{B}_{\mathbb{O}}$, we observe that $A=B^{I}$ is the set of all $x \in X$ such that $\bigwedge B$ is an upper bound of $F(x)$. Also, for convenience, for a node $p \in P$ denote $p^{I}:=\{p\}^{I} \subseteq X$.

\section{$3 \quad$ Pattern Structures}

In KG01 pattern structures are introduced to model information in the realm of pharmaceutical research. How pattern structures serve to analyze many-valued data contexts is described in $\mathrm{Ku} 09$.

Definition 2 (pattern structure). Let $G$ be a set, let $\mathcal{D}:=(D, \sqcap)$ be a meetsemilattice, and let $\delta: G \rightarrow D$. Then $\mathbb{P}:=(G, \mathcal{D}, \delta)$ is called pattern structure if $\delta(G)$ generates a complete subsemilattice $\left(D_{\delta}, \sqcap\right)$ of $\mathcal{D}$. The elements of $G$ are called objects and the elements of $D$ are called descriptions or patterns. The operation $\sqcap$ models a similarity operation on the descriptions.

Pattern structures can be represented by the concept lattices of so called representation contexts. In the following we sketch the construction of representation contexts as given in KG01. First it is important to note that $\left(D_{\delta}, \sqcap\right)$ is complete and therefore, there exists a (unique) operation $\sqcup$ to make $\left(D_{\delta}, \sqcap, \sqcup\right)$ into a complete lattice. This operation (on $D$ ) is given by $\bigsqcup X:=\prod\left\{c \in D_{\delta} \mid \forall x \in\right.$ $X: x \sqsubseteq c\}$. Furthermore, a subset $M$ of $D$ is called $\sqcup$-dense for $\left(D_{\delta}, \sqcap\right)$ if any element $d \in D_{\delta}$ can be recaptured as join of elements $N \subseteq M$, that is $d=\bigsqcup N$. Now $(G, M, I)$ is a representation context for $(G, \mathcal{D}, \delta)$ if $M$ is $\sqcup$-dense for $\left(D_{\delta}, \sqcap\right)$ and $I \subseteq G \times M$ is defined as $g I m: \Longleftrightarrow \delta(g) \sqsupseteq m$. 


\section{Basic Connections}

On the one hand, given an elementary annotated ordered set $\mathbb{O}:=(\mathcal{P}, X, F)$ we get a pattern structure $\mathbb{O}:=\left(X, \mathcal{P}_{\sqcap}, F\right)$ if $\mathcal{P}$ allows for (finite) meets and $F(X)$ generates a complete subsemilattice of $\mathcal{P}_{\sqcap}$. On the other hand, given a pattern structure $\mathbb{P}:=(G, \mathcal{D}, \delta)$ we always get an elementary annotated ordered set $\left(\mathcal{D}_{\sqsubseteq}, G, \delta\right)$ when considering the meet-semilattice as ordered set via the usual definition $c \sqsubseteq d: \Leftrightarrow c \sqcap d=c$. Therefore, we see that pattern structures are special cases of annotated ordered sets and we can immediately transfer all results on annotated ordered sets to pattern structures. When we consider the other direction we must be more careful. But let us first compare the constructions of formal contexts for representation in both cases.

For the time being, let us consider a pattern structure $\mathbb{P}:=(G, \mathcal{D}, \delta)$ as elementary annotated ordered set and let us build its associated context via the annotated ordered sets method. We get $\mathbb{K}_{\mathbb{P}}:=(G, D, I)$ where $I \subseteq G \times D$ is given by $g I d: \Leftrightarrow \delta(g) \sqsubseteq d$. It is obvious that $D$ is $\sqcup$-dense for $D_{\delta}$. If we had dualized the order $\sqsubseteq$ upfront the above construction would have yielded a representation context for $\mathbb{P}$. Therefore, we can conclude that the method for representation context construction for pattern structures generalizes the method for concept lattice representation for annotated ordered sets in this case. It is important to note that Theorem 1 in KG01 implies that the concept lattices of (different) representation contexts for a given pattern structure are isomorphic.

In [KG01, it follows directly from Theorem 1 that $\left(D_{\delta}, \sqsubseteq\right)$ and the concept lattice of a representation context of the underlying pattern structure are antiisomorphic. In the following, we exhibit a connection between the concept lattice of a representation context and $\mathcal{D}$, the ordered set of all patterns. For elementary annotated complete lattices, Theorem 1 in [KSJ08 makes obvious that their concept lattice representations are tied to the underlying complete lattices via adjunctions. We need to generalize this result to pattern structures, since $(D, \sqsubseteq)$ is not necessarily a complete lattice.

Theorem 1. Let $\mathbb{P}:=(G, \mathcal{D}, \delta)$ be a pattern structure, let $\mathbb{K}:=(G, M, I)$ be a representation context, let $\varphi: \mathfrak{B}(\mathbb{K}) \longrightarrow D,(A, B) \mapsto \sqcup B$ be a mapping, and let $\mu: D \longrightarrow \mathfrak{B}(\mathbb{K}), d \mapsto\left(d^{I}, d^{I I}\right)$ be a mapping. Then $(\varphi, \mu)$ forms a dual adjunction (or Galois connection) between the ordered set of patterns $\mathcal{D}$ and the concept lattice $(\mathfrak{B}(\mathbb{K}), \leq)$ of $(G, M, I)$. In particular, $\varphi$ is injective and $\mu$ is surjective.

Proof. We show that $\varphi$ is injective (the surjectivity of $\mu$ follows from the injectivity of $\varphi$ ). Assume we have two concepts $(A, B)$ and $(C, D)$ which are mapped to the same element, that is, $\sqcup B=\sqcup D$. We will show that $(\sqcup B)^{I}=A$ from which it follows that $(A, B)=(C, D)$. We have that $g \in(\sqcup B)^{I}$ if and only if $\delta(g) \sqsupseteq \sqcup B$ if and only if $\delta(g) \sqsupseteq b$ for all $b \in B$ if and only if $g \in B^{I}$.

The pair $(\varphi, \mu)$ forms a Galois connection since $\varphi(A, B) \sqsubseteq d \Longleftrightarrow \sqcup B \sqsubseteq d \Longleftrightarrow$ $(\sqcup B)^{I} \supseteq d^{I} \Longleftrightarrow(A, B) \geq\left(d^{I}, d^{I I}\right)=\mu(d)$

From the above theorem it follows that the concept lattice of a representation context is embedded as a closure system into $\mathcal{D}$. 
In KSJ08, it is investigated what can happen if we fix an ordered set and then vary the annotations. It turns out that the annotations are in one-to-one correspondence with the closure systems in the filter lattice of the ordered set. Clearly, this implies that in the case of pattern structures if a set of patterns or descriptions together with a similarity operation is given and we vary the annotation function $\delta$ freely, we can only get a one-to-one correspondence between (elementary) annotations and a certain subset of the closure systems in the ideal lattice (the dual of the filter lattice of the ordered set). In the following we will single out this subset.

Theorem 2. Let $G$ be a set of objects, and let $\mathcal{D}:=(D, \sqcap)$ be a semi-lattice. The elementary annotations are, up to annotational equivalence, in one-to-one correspondence to the principally generated closure systems in the ideal lattice of $\mathcal{D}$.

Proof. Given an elementary annotation $\delta: G \rightarrow D$ every $x^{\delta \circ \sqsupseteq}$ is a principal ideal in $\mathcal{D}_{\sqsubseteq}$ and an object intent of a formal concept of $(G, D, \delta \circ \sqsupseteq)$. Since, in general, the intents of all concepts of a formal context are exactly the meets of the object intents the annotation induces a principally generated closure system in the ideal lattice of $\mathcal{D}$.

Given a closure system $\mathcal{X} \subseteq 2^{D}$ in the ideal lattice of $\mathcal{D}_{\sqsubseteq}$ generated by the principal ideals $\mathcal{I} \subseteq 2^{D}$ we can define a corresponding elementary annotation $\delta_{\mathcal{I}}: \mathcal{I} \rightarrow D$ where $\downarrow i \mapsto i$.

\section{Projections}

For pattern structures so called projections where introduced in [KG01] to model the process of simplification of the underlying subsumption order. Projections are defined as kernel operator 11 on the ordered set of patterns or descriptions. The existence of projections can be characterized by the existence of certain representation contexts. We quote Theorem 2 from [KG01]

For pattern structures $\mathbb{P}_{1}:=\left(G, \mathcal{D}, \delta_{1}\right)$ and $\mathbb{P}_{2}:=\left(G, \mathcal{D}, \delta_{2}\right)$ the following two conditions are equivalent.

(1) There exists a projection $\pi$ with $\delta_{2}=\delta_{1} \circ \pi$.

(2) There exist representation contexts $(G, M, I)$ of $\mathbb{P}_{1}$ and $(G, N, I \cap(G \times N))$ of $\mathbb{P}_{2}$ with $N \subseteq M$.

In the proof of the theorem it is claimed that $\pi(d):=\bigsqcup\{n \in N \mid n \sqsubseteq d\}$ is a kernel operator on $D$ if $(G, N, I)$ is a representation context of $(G, \mathcal{D}, \delta)$. Examples 1 shows that condition (2) in Theorem 2 needs to be boosted 2 and Example 2 shows that the above operator used in the proof is not a kernel operator as claimed.

\footnotetext{
${ }^{1}$ Kernel operators on ordered sets are isotone, idempotent, and dually extensive self-maps.

2 Thanks to Jan Frebel for valuable input.
} 
Example 1. Let $\mathcal{D}$ be given by the structure depicted in Figure 1 and let $G_{i}:=$ $\{g\}$ and $\delta_{i}(g):=a_{i}$ and $M_{i}:=\{0, c\}$ (visualized by the dotted compartment in Figure1) for $i=1,2$. Note that the carrier set $D_{\delta}$ of the complete sub-semilattice generated by $\delta(G)$ is given by $\left\{a_{i}, 1\right\}$ for $i=1,2$. It is immediate that $M_{i}$ is $\sqcup$ dense regarding $\delta_{i}$ for $i=1,2$ and that $M_{2} \subseteq M_{1}$, but obviously there exists no projection $\pi$ with $\delta_{2}=\delta_{1} \circ \pi$.

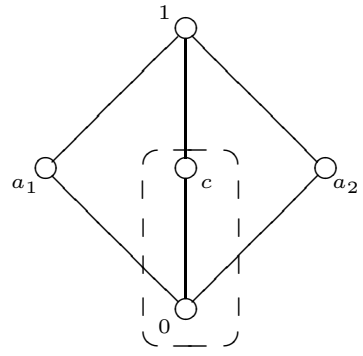

Fig. 1. (2) implies (1) does not hold in Theorem 2

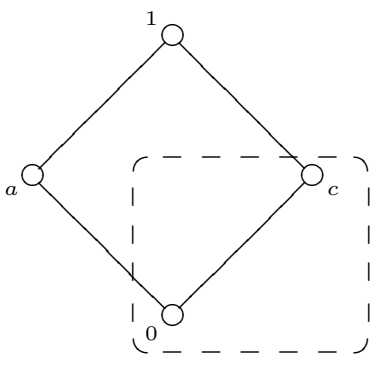

Fig. 2. Operator from the proof is not a kernel operator

Example 2. Let $\mathcal{D}$ be given by the structure depicted in Figure 2 and let $G:=\{g\}$ and $\delta(g):=a$ and $M:=\{0, c\}$ (visualized by the dotted compartment in Figure 2). Note that the carrier set $D_{\delta}$ of the complete sub-semilattice generated by $\delta(G)$ is given by $\{a, 1\}$. It is immediate that $M$ is $\sqcup$-dense regarding $\delta$, but $\pi: D \rightarrow D: d \mapsto \sqcup\{m \in M \mid m \sqsubseteq d\}$ is not contractive since $\pi(c)=1$, thus $\pi$ is not a kernel operator.

To repair Theorem 2 we additionally require that $\delta_{2}$ is point-wise contained in $\delta_{1}$ in condition (2) and to repair the proof we make the above operator into a kernel operator.

Theorem 3. For pattern structures $\mathbb{P}_{1}:=\left(G, \mathcal{D}, \delta_{1}\right)$ and $\mathbb{P}_{2}:=\left(G, \mathcal{D}, \delta_{2}\right)$ the following two conditions are equivalent.

(1) There exists a projection $\pi$ with $\delta_{2}=\delta_{1} \circ \pi$.

(2) We have $\delta_{2} \sqsubseteq \delta_{1}$ and there exist representation contexts $(G, M, I)$ of $\mathbb{P}_{1}$ and $(G, N, I \cap(G \times N))$ of $\mathbb{P}_{2}$ with $N \subseteq M$.

Proof. " $(1) \Rightarrow(2)$ ": Since there exists a projection $\pi$ with $\delta_{2}=\delta_{1} \circ \pi$ we have for $d \in D$ that $\delta_{2}(d)=\delta_{1} \circ \pi(d)=\pi\left(\delta_{1}(d)\right)$ which implies $\delta_{2}(d) \sqsubseteq \delta_{1}(d)$ since $\pi$ is contractive. We construct the two representation contexts. Let $M:=D$ and let $N:=\pi(D)$. Obviously, we have $N \subseteq M$. Since $\delta_{2}=\delta_{1} \circ \pi$ we get $\delta_{2}(G) \subseteq \pi(D)$. It remains to show that arbitrary meets of elements from $\delta_{2}(G)$ can be recovered from $N$. Assume $F \subseteq \delta_{2}(G)$ and let $f:=\prod F$. Since $\delta_{2}(G) \subseteq \pi(D)$ and $\pi$ is idempotent we have $F=\pi(F)$ which yields $f=\prod \pi(F)$. Since kernel operators 
are (completely) meet-preserving we get $f=\prod \pi(F)=\pi\left(\prod F\right) \in \pi(D)$. That means $D_{\delta_{2}} \subseteq \pi(D)$ which shows that $\pi(D)$ is $\sqcup$-dense regarding $D_{\delta_{2}}$. The relation $I$ of the first representation context is given by $g I d: \Leftrightarrow \delta_{1}(g) \sqsupseteq d$. If we look at the relation $J$ of the second representation we see that for $d \in \pi(D)$ we have $g J d: \Leftrightarrow \delta_{2}(g) \sqsupseteq d$. But since $\pi$ is a kernel operator for $d \in \pi(D)$ it follows that $\delta_{2}(g) \sqsupseteq d \Longleftrightarrow \pi\left(\delta_{2}(g)\right) \sqsupseteq \pi(d) \Longleftrightarrow \pi\left(\delta_{2}(g)\right) \sqsupseteq d \Longleftrightarrow \delta_{1}(g) \sqsupseteq d$ which yields $J=I \cap(G \times \pi(D))$.

"(2) $\Rightarrow(1) "$ : We define a self-map on $D$ by $\pi(d):=d \sqcap \bigsqcup\{n \in N \mid n \sqsubseteq$ $d\}=d \sqcap \bigsqcup \downarrow_{N} d$. Note that, in general, $\sqcup$ is not the supremum operation for $D$. Therefore, we must be extra-cautious while showing that $\pi$ is a kernel operator. It is easy to see that $\pi$ acts on $D_{\delta_{2}}$ as the identity since $N$ is $\sqcup$-dense regarding $D_{\delta_{2}}$. Also, by definition of $\bigsqcup$, the image of $\pi$ equals $D \cap D_{\delta_{2}}$. For showing that $\pi$ is monotone, we assume that $d \leq d^{\prime}$. We have $\downarrow_{N} d \subseteq \downarrow_{N} d^{\prime}$ which implies that $\left\{c \in D_{\delta} \mid \forall x \in \downarrow_{N} d^{\prime}: x \sqsubseteq c\right\}$ is a subset of $\left\{c \in D_{\delta} \mid\right.$ $\left.\forall x \in \downarrow_{N} d: x \sqsubseteq c\right\}$ and therefore $\bigsqcup \downarrow_{N} d=\prod\left\{c \in D_{\delta} \mid \forall x \in \downarrow_{N} d: x \sqsubseteq c\right\}$ is less or equal than $\sqsubseteq \prod\left\{c \in D_{\delta} \mid \forall x \in \downarrow_{N} \quad d^{\prime}: x \sqsubseteq c\right\}=\bigsqcup \downarrow_{N} d^{\prime}$. This implies $\pi(d)=d \sqcap \bigsqcup \downarrow_{N} d \sqsubseteq d \sqcap \bigsqcup \downarrow_{N} d^{\prime}=\pi\left(d^{\prime}\right)$ as required. Contractivity of $\pi$ is immediate. It remains to show idempotency. It suffices to make sure that $\downarrow_{N} \pi(d)=\downarrow_{N} d$. Since $\pi$ is contractive we have $\downarrow_{N} \pi(d) \subseteq \downarrow_{N} d$. Let $n \in N$ with $n \sqsubseteq d$. Obviously $n \sqsubseteq d \sqcap \bigsqcup \downarrow_{N} d=\pi(d)$. We get $\downarrow_{N} \pi(d) \supseteq \downarrow_{N} d$ which gives $\downarrow_{N} \pi(d)=\downarrow_{N} d$. We have shown that $\pi$ is a kernel operator on $D$. It remains to show that $\delta_{2}=\delta_{1} \circ \pi$ holds. Let $g \in G$ and $n \in N$. From condition (2) it follows that $n \sqsubseteq \delta_{1}(g) \Leftrightarrow g I n \Leftrightarrow g\left(I \cap(G \times N) n \Leftrightarrow n \sqsubseteq \delta_{2}(g)\right.$. We deduce $\sqcap \bigsqcup \downarrow_{N}$ $\delta_{1}(g)=\sqcap \bigsqcup \downarrow_{N} \delta_{2}(g)$. Expanding the definitions and using the above equations we get $\pi\left(\delta_{1}(g)\right)=\delta_{1}(g) \sqcap \bigsqcup \downarrow_{N} \delta_{1}(g)=\delta_{1}(g) \sqcap \bigsqcup \downarrow_{N} \delta_{2}(g)=\delta_{1}(g) \sqcap \delta_{2}(g)$. Since we assume $\delta_{1} \sqsubseteq \delta_{2}$ we have $\delta_{1}(g) \sqcap \delta_{2}(g)=\delta_{2}(g)$ and deduce $\left(\delta_{1} \circ \pi\right)(g)=\delta_{2}(g)$ to complete our proof.

\section{References}

[DP90] Davey, B.A., Priestly, H.A.: Introduction to Lattices and Order. Cambridge University Press, Cambridge (1990)

[GW99] Ganter, B., Wille, R.: Formal Concept Analysis. Mathematical Foundations. Springer, Berlin Heidelberg New York (1999)

[KSJ08] Kaiser, T.B., Schmidt, S.E., Joslyn, C.A.: Adjusting Annotated Taxonomies. International Journal of Foundations of Computer Science (IJFCS): Special Issue: Concept Lattices and Their Applications 18(2), 345358 (2008)

[KG01] Ganter, B., Kuznetsov, S.O.: Pattern Structures and Their Projections. In: Delugach, H.S., Stumme, G. (eds.) ICCS 2001. LNCS (LNAI), vol. 2120, pp. 129-142. Springer, Heidelberg (2001)

[Ku09] Kuznetsov, S.O.: Pattern Structures for Analyzing Complex Data. In: Sakai, H., Chakraborty, M.K., Hassanien, A.E., Ślęzak, D., Zhu, W. (eds.) RSFDGrC 2009. LNCS, vol. 5908, pp. 33-44. Springer, Heidelberg (2009) 